\title{
Conditional switching of VEGF provides new insights into adult neovascularization and pro-angiogenic therapy
}

\author{
Yuval Dor, Valentin Djonov', \\ Rinat Abramovitch ${ }^{2}$, Ahuva Itin, \\ Glenn I.Fishman ${ }^{3}$, Peter Carmeliet ${ }^{4}$, \\ Gadi Goelman ${ }^{1}$ and Eli Keshet ${ }^{5}$
}

Department of Molecular Biology, Hebrew University-Hadassah Medical School, ${ }^{2}$ MRI/MRS Laboratory, HBRC, Hadassah University Hospital, Jerusalem 91120, Israel, ${ }^{1}$ Department of Anatomy, Berne University, Switzerland, ${ }^{3}$ Section of Myocardial Biology, Department of Medicine, Mount Sinai School of Medicine, New York, NY, USA and ${ }^{4}$ The Center for Transgene Technology and Gene Therapy, Flanders Interuniversity Institute for Biotechnology, KU Leuven, B-3000 Leuven, Belgium

${ }^{5}$ Corresponding author

e-mail: keshet@cc.huji.ac.il

To gain insight into neovascularization of adult organs and to uncover inherent obstacles in vascular endothelial growth factor (VEGF)-based therapeutic angiogenesis, a transgenic system for conditional switching of VEGF expression was devised. The system allows for a reversible induction of VEGF specifically in the heart muscle or liver at any selected schedule, thereby circumventing embryonic lethality due to developmental misexpression of VEGF. Using this system, we demonstrate a progressive, unlimited ramification of the existing vasculature. In the absence of spatial cues, however, abnormal vascular trees were produced, a consequence of chaotic connections with the existing network and formation of irregularly shaped sac-like vessels. VEGF also caused a massive and highly disruptive edema. Importantly, premature cessation of the VEGF stimulus led to regression of most acquired vessels, thus challenging the utility of therapeutic approaches relying on short stimulus duration. A critical transition point was defined beyond which remodeled new vessels persisted for months after withdrawing VEGF, conferring a long-term improvement in organ perfusion. This novel genetic system thus highlights remaining problems in the implementation of pro-angiogenic therapy.

Keywords: angiogenesis/conditional transgene/therapy/ VEGF

\section{Introduction}

Extensive formation of blood vessels takes place in the developing embryo, guided by stereospecific developmental cues. In the adult, neovascularization is mostly confined to the female reproductive system but is also induced in response to perturbations in oxygen homeostasis, primarily following occlusion of major blood vessels. Ischemiadriven neovascularization mediated by vascular endothelial growth factor (VEGF) (Dor and Keshet, 1997), acting together with a vessel remodeling process termed arteriogenesis (van Royen et al., 2001), operates to improve the perfusion of ischemic tissues. In most clinical settings, however, these natural adaptive responses to a compromised perfusion are insufficient and unable to halt the progression of an ischemic disease in the myocardium or in peripheral vessels. The reasoning behind proangiogenic therapy for ischemic diseases is that exogenous administration of an angiogenic factor(s) might augment the natural collateralization process to an extent that the need for a more drastic (e.g. surgical) intervention will be circumvented. Indeed, encouraging pre-clinical studies have already prompted clinical trials of pro-angiogenic therapy (for recent reviews on pre-clinical studies and clinical trials see Hammond and McKirnan, 2000; Simons et al., 2000; Freedman and Isner, 2001). However, there are growing concerns that the large gaps that exist in our knowledge regarding the fundamentals and mechanisms of neovascularization of adult organs jeopardize favourable outcomes of these trials (Carmeliet, 2000; Helisch and Schaper, 2000; Lee et al., 2000; Blau and Banfi, 2001; Laham et al., 2001).

Several factors regarding adult neovascularization need to be considered with therapeutic angiogenesis. First, most contemplated pro-angiogenic therapies rely on the administration of a single factor, whereas the natural process is multi-factorial. As an illustration, precluding signaling through each of the five endothelial cell-specific tyrosine kinase receptors (VEGF-R1, VEGF-R2, VEGF-R3, Tie1 and Tie2) results in an abortive vessel formation and embryonic lethality (Gale and Yancopoulos, 1999). Furthermore, additional factors and signaling systems are essential for engagement with periendothelial cells [e.g. the plateletderived growth factor (PDGF) system; Lindahl et al., 1997] and others still are required for specifying an arterial or a venous fate (e.g. ephrin-B2/ephrin-B4; Adams and Klein, 2000). The premise that a singly administered factor is capable of inducing all other complementary activities essential for formation of a fully functional network needs to be substantiated and the quality of induced vessels carefully examined. Secondly, it remains to be determined how such a complex morphogenetic process is executed in the absence of guiding cues and under circumstances where neither the normal dosage nor the spatial or temporal controls of the initiating morphogen are maintained. Improper connectivity might perturb rather than improve the coronary circulation. Thirdly, it has to be determined whether short-term therapy will produce stable vessels that will persist after withdrawal of the inductive stimulus. This issue is of particular concern in light of earlier studies showing that premature withdrawal of VEGF may lead to regression of immature vessels in settings of developmental and tumor angiogenesis (Alon et al., 1995; Benjamin and Keshet, 1997).

Addressing these fundamental issues in pre-clinical studies has been hampered by several problems. 
Depending on the methodology used for growth factor delivery, complications may include insufficient or variable levels of VEGF in the target organ, 'off target' effects, insufficient duration of the stimulus, an accompanying inflammatory response and an immunological reaction. Thus, it has been difficult to determine whether a particular shortcoming is due to any of these external factors or, alternatively, due to a fundamental problem inherent to a single factor-based therapeutic angiogenesis in the adult. We sought an experimental genetic system that would circumvent the above shortcomings and allow evaluation of the performance of a particular growth factor under optimal conditions. To this end, a genetic switch was designed in a transgenic mouse system that allowed for the reversible induction of high levels of VEGF, the factor most frequently considered for therapeutic angiogenesis, exclusively at the selected target organ and was able to maintain the stimulus for the desired duration. The resultant acquired vascular network was analyzed structurally by visualizing its three-dimensional architecture and network connectivity with the aid of corrosion casting, and functionally by monitoring dynamic changes in blood oxygenation and tissue edema using magnetic resonance imaging (MRI). The results reported below, while demonstrating the promise of a VEGF-based therapy, also uncover some inherent obstacles that need to be resolved first.

\section{Results}

\section{A transgenic mouse system for conditional switching of VEGF expression in selected organs}

To evaluate the feasibility of a VEGF-based pro-angiogenic therapy, we designed an animal model system in which a source of VEGF can be specifically induced in the target organ, steadily maintained for the desired duration and subsequently switched off. Considering previous findings that a slight deviation from the normal dosage of VEGF results in embryonic lethality (Carmeliet et al., 1996; Ferrara et al., 1996; Miquerol et al., 2000), we resorted to a conditional transgenic system for VEGF induction.

Transgenic mice expressing a tetracycline-regulated transactivator protein (tTA) exclusively in the myocardium or liver were employed as 'driver lines'. Another transgenic line harboring a VEGF164-encoding transgene (driven by a tetracycline-responsive promoter) was used as the 'responder line'. The respective 'driver' mouse was mated with the VEGF 'responder' animal, and pups that inherited both transgenes were selected (see scheme in Figure 1A). The onset of VEGF induction in these animals and the duration of expression were tightly controlled through the inclusion or omission of tetracycline from the drinking water ('off' and 'on' modes, respectively). Littermates that inherited only one of the two transgenes and were concomitantly subjected to the same repression/induction regime served as controls. Heart-specific induction was achieved using a driver line in which tTA expression is driven by a myosin heavy chain- $\alpha(\mathrm{MHC} \alpha)$ heart-specific promoter, previously shown to be expressed specifically by cardiomyocytes (Yu et al., 1996). Liver-specific induction was obtained by using a driver line in which tTA expression is driven by a
CCAAT/enhancer binding protein $\beta(\mathrm{C} / \mathrm{EBP} \beta)$ promoter (also known as liver activator protein or PLAP), expressed primarily in hepatocytes (Kistner et al., 1996). If kept unrepressed, misexpression of VEGF in either organ led to multiple developmental defects and embryonic lethality (Dor et al., 2001; our unpublished results). Furthermore, if repression was relieved during the first 2-3 weeks after birth (when extensive growth in either organ is still ongoing), death also ensued. In contrast, in the presence of tetracycline, $\mathrm{tTA}^{+} / \mathrm{VEGF}^{+}$animals were born with the expected frequency and if VEGF induction was delayed until later than 3 weeks after birth there was no lethality.

In $\mathrm{tTA}^{+} / \mathrm{VEGF}^{+}$animals VEGF164 expression was strongly induced upon withdrawal of tetracycline. In situ hybridization confirmed that when driven by MHC $\alpha$-tTA, expression of VEGF was induced in most, if not all, cardiomyocytes (Figure 1B) and when driven by $\mathrm{P}_{\mathrm{LAP}}$ tTA, in most, if not all, hepatocytes (Figure 1C). This was assurance that extensive areas within the respective organ would be affected by the secreted factor.

Quantitatively, the level of VEGF expression in the myocardium was increased by $\sim 7$-fold overall (Figure $1 \mathrm{~B}$ ) and the level of VEGF in the liver was increased by $\sim 11$-fold compared with the level of endogenous VEGF (Figure 1C). The continuous production of VEGF in the liver also resulted in its accumulation in the serum to levels that are 5- to 10-fold higher than the normal level of $300 \mathrm{pg} / \mathrm{ml}$, as determined by a VEGF enzyme-linked immunosorbent assay (ELISA). In contrast, we could not detect a significant elevation of VEGF in the serum with cardiac-specific overexpression.

Further evidence that the system is tightly controlled and fully reversible is provided by the northern blot of a representative 'liver switch' experiment shown in Figure 1C. The transgenic VEGF allele is completely silent prior to the switch and is effectively re-repressed upon re-administration of tetracycline.

\section{VEGF induces unlimited formation of new blood vessels in the adult myocardium and liver}

To determine whether VEGF can promote the formation of new blood vessel in adult tissues when acting alone, VEGF expression was switched 'on' in the myocardium or liver of adult animals. Organs were retrieved at different times following a continuous exposure to the VEGF stimulus and their vasculature was analyzed.

Shortly after inducing VEGF in the heart, there was a massive accumulation of cells interspersed in between the muscle fibres. These cells were tentatively assigned as endothelial precursor cells (EPCs) on the basis of expressing a panel of endothelial cell-specific markers (the characterization of EPCs and their possible origins will be described elsewhere; Y.Dor and E.Keshet, in preparation). Coalescence of these cells into discrete blood-filled vessels was subsequently apparent. Notably, new vessels continued to be formed as long as the VEGF stimulus was present. The continuous nature of the process was also apparent from experiments using short (3 h) BrdU pulses to tag endothelial cells in $\mathrm{S}$ phase, demonstrating a significant fraction of cycling endothelial cells at any time point during a 1-2 month course of VEGF induction (data not shown). Prolonged VEGF stimulus in the myocardium resulted in a very high vessel density and in formation of 
A

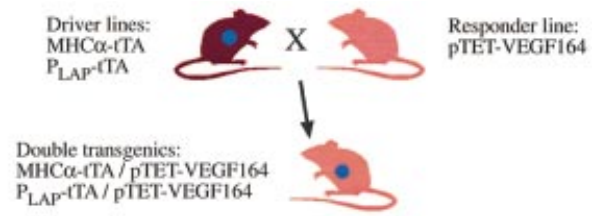

B Heart switch
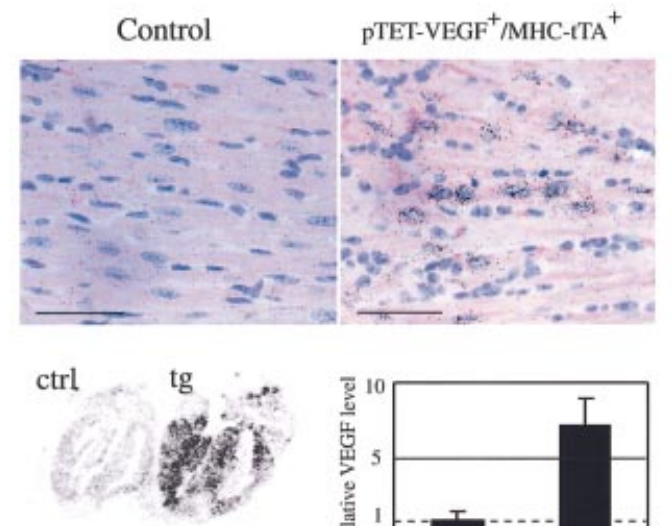

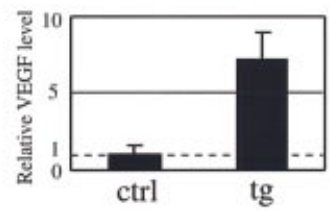

\section{Liver switch}
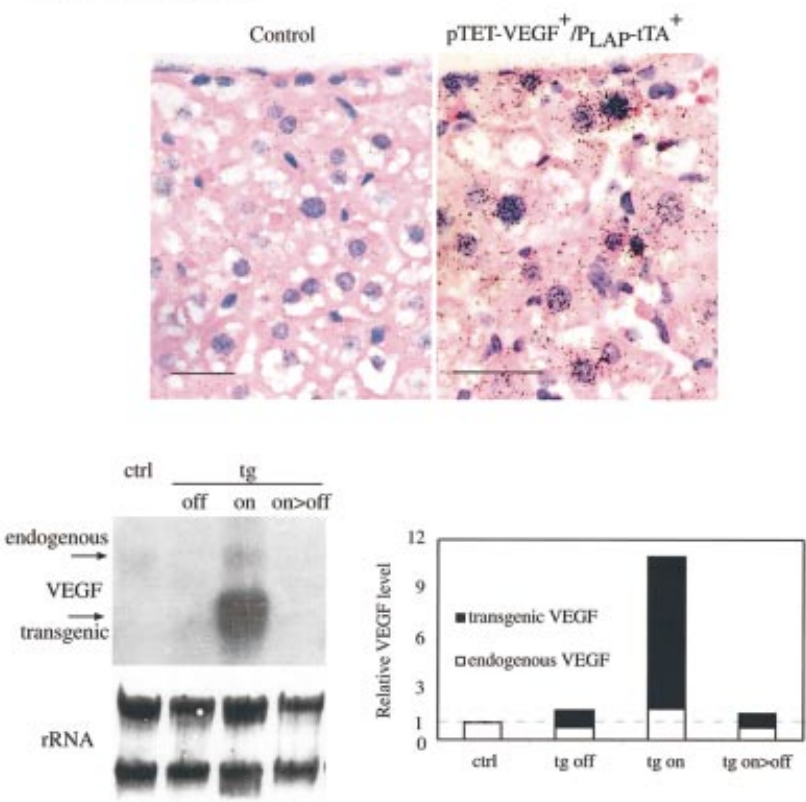

Fig. 1. A transgenic mouse system for conditional switching of VEGF expression in the adult heart or liver. (A) Scheme of the binary transgenic system based on a tetracycline-regulated trans-activator protein driven by either a heart- or liver-specific promoter ('driver' lines) and a transgene encoding VEGF164 ('responder' line) (see text for details). (B) A heart-specific VEGF switch. VEGF expression was induced by withdrawing tetracycline 1 week after birth and retrieving hearts for analysis 3 weeks later. Upper panels show in situ hybridization with a VEGF probe using myocardial thin sections obtained from littermates control and double transgenic animals that have been processed and hybridized on the same slide. Note induced expression of VEGF in cardiomyocytes. Myocardial hypercellularity observed in the double transgenic heart is due to VEGF-mediated proliferation/recruitment of endothelial cell precursors. Bars, $50 \mu \mathrm{m}$. Bottom left: a low magnification view of heart sections hybridized with VEGF as above but autoradiographed through a direct contact with an X-ray film to highlight the overall distribution of VEGF-producing cells. Bottom right: Relative levels of VEGF expression in myocardial sections quantified by autogradiographic grains counts (average \pm SD of three high-power fields from each of three independent hybridizations). (C) A liver-specific VEGF switch. VEGF expression was induced by withdrawing tetracycline 1 week after birth and retrieving livers for analysis 4 weeks later. Upper panels show in situ hybridization with a VEGF probe using hepatic thin sections obtained from littermates control and double transgenic animals that have been processed and hybridized on the same slide. Note induced expression of VEGF in hepatocytes. Bars, $50 \mu \mathrm{m}$. Bottom left: northern blot analysis of total liver RNA extracted from control animals and from double transgenic animal prior to tetracycline withdrawal (off), 1 month after an 'on' switch (on) and 1 month after an 'on' switch followed by 3 months in the presence of tetracycline (on>off). Bottom right: Relative levels of VEGF expression were determined by densitometric tracing of the autoradiogram distinguishing the endogenous from transgenic VEGF.

vessels with abnormally large lumens occupying a large fraction of the organ (Figure 2A). Thus, VEGF-promoted neovascularization in the adult myocardium does not appear to be a self-limiting process and is also not restricted by the exhaustion of some needed downstream factors.

To determine whether VEGF can promote formation of large coronary vessels coated with vascular smooth muscle cells (VSMCs), myocardial sections were immunostained with the SMC/pericyte-specific marker $\alpha$-smooth muscle actin $(\alpha \mathrm{SMA})$. As shown in Figure $2 \mathrm{~B}$ (low magnification) and in Figure 2C (high magnification), VEGF indeed promoted formation of large coronary vessels, a fraction of which are coated with smooth muscle cells. This result indicates that VEGF not only promotes formation of an endothelial plexus but also supports the recruitment of periendothelial cells, presumably by inducing other activities required to accomplish this function. This finding provides further support for the concept of a single factorbased pro-angiogenic therapy. As is evident from Figure 2C, VEGF also caused extensive edema, completely disrupting the otherwise tight muscle architecture.
Parallel experiments using a liver-specific VEGF switch demonstrated that VEGF also induced robust liver neovascularization. The elaborate vascular network induced by VEGF is clearly visible by external inspection (Figure 2D). Notably, the induced network is properly connected to the hepatic circulation, as evidenced by the fact that it carries blood. Histological examination has shown that vessels induced by VEGF in the liver are mostly large sinusoidal vessels (Figure 5A).

\section{Abnormal structure and connectivity of an induced myocardial network}

For optimal functioning, new vessels induced by VEGF must not only form a well-graded vascular tree but also appropriately connect with the existing coronary network. To visualize the three-dimensional architecture of the induced coronary network, corrosion casts were prepared and inspected by scanning electron microscopy. This methodology visualizes the entire vascular tree at a resolution that includes even the smallest capillaries. Since it detects only perfused vessels accessible to the casting resin, it highlights only functional vascular loops 

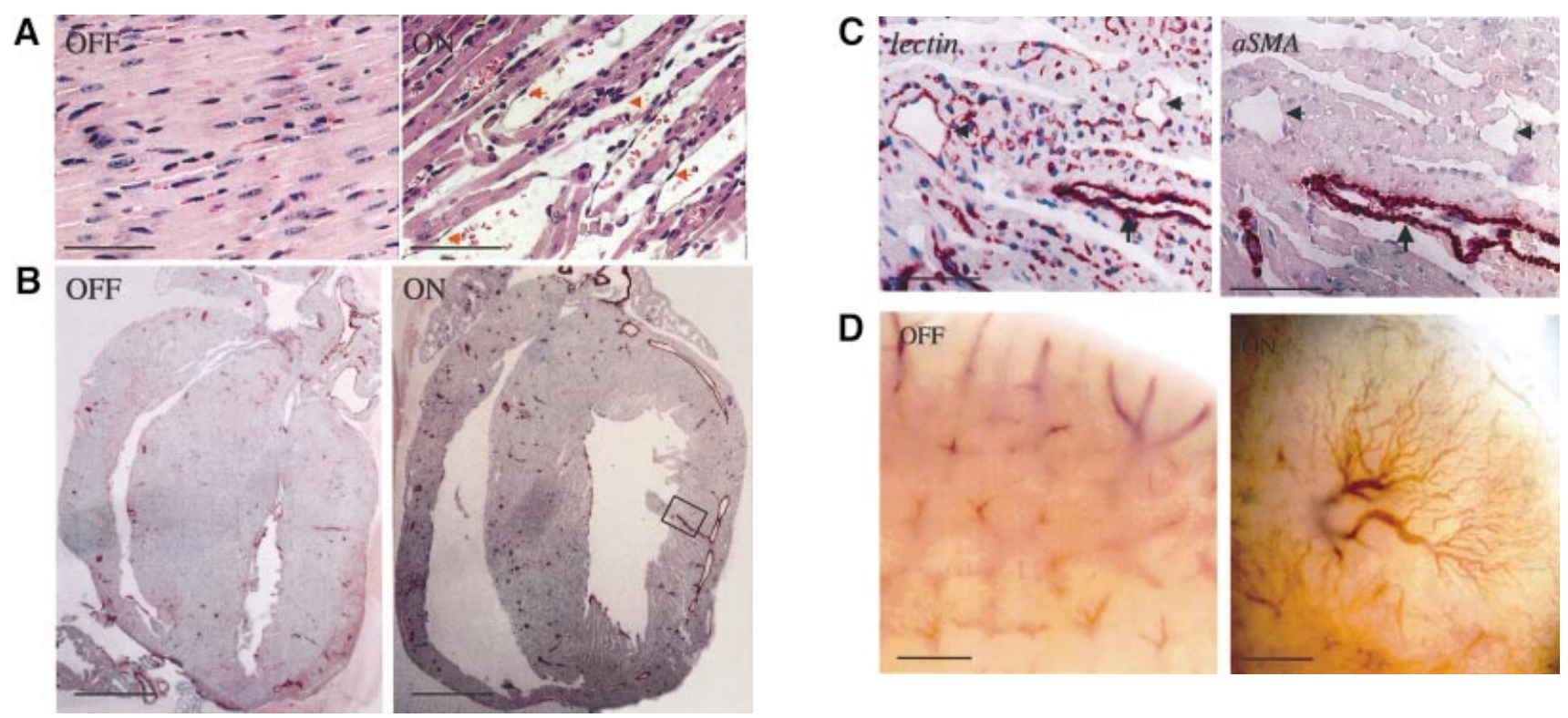

Fig. 2. VEGF induces extensive vessel formation in the adult myocardium and liver. (A) Hematoxylin-eosin staining of myocardial sections following a 19-day myocardial VEGF switch. An exaggerated angiogenic response has produced many vessels inter-digitizing muscle fibres and perturbing their normal architecture. Arrows point to the endothelium of these vessels. Bar, $100 \mu \mathrm{m}$. (B) A low-power view of the myocardium following a myocardial VEGF switch. Immunostaining with $\alpha$ SMA highlights large coronary vessels covered by VSMCs and pericytes. Note the higher abundance of $\alpha$ SMApositive vessels in the VEGF-induced myocardium compared with control (quantified as a 2.4-fold increase through counting $\alpha$ SMA-positive vessels in the entire ventricular wall in five sections each). Bar, $1 \mathrm{~mm}$. (C) Right: a higher magnification of the area boxed in (B). Note the presence of both VSMC-coated vessels (arrow) as well as uncoated vessels (arrowheads). Left: immunostaining with an endothelial cell-specific lectin (Bandeiraea simplicifolia BS-1 isolectin) performed on a serial section. Staining appearing in single cells presumably represent sections through capillaries as well as endothelial precursor cells. Bars, $100 \mu \mathrm{m}$. (D) Whole-mount views of a liver following a 30-day switch (a bottom view at the edge of the lobes). Note the elaborate vascular tree induced by VEGF. Bar, $1 \mathrm{~mm}$.

connected to the systemic circulation. A typical segment of the myocardial vascular tree in control heart is shown in Figure 3A. Switching 'on' VEGF expression and maintaining the stimulus for several weeks led to progressive ramification of the vasculature. Initially, this was evident mostly by reduced inter-capillary distance, and by structures suggestive of ongoing fusion events between contacting small vessels (see Supplementary figure 1 available at The EMBO Journal Online). Further progression of the process during later stages resulted in the generation of very large vessels and eventually in the production of hemangioma-like vascular sacs (see Figure 3B for a representative example). Some of the vessels shown in Figure 3B seem to be in the process of flattening on top and merging their lumens with those of existing larger vessels (arrows). Abnormal structural features of these vessels included a variable diameter in consecutive segments within a single vessel, a large proportion of vessels with multiple rather than bimodal branching points (arrowhead) and abnormal branching angularities (Figure 3B). Thus, it appears that in the absence of guiding cues, vessels induced by VEGF in the adult myocardium connect with the existing coronary network in a highly chaotic manner. Interestingly, following VEGF withdrawal, the network resumed a more 'normal' appearance (Figure 3C; discussed below).

\section{Vessels induced by VEGF improve organ perfusion but are leaky: an MRI analysis}

To determine whether the vascular gain resulting from a sustained VEGF stimulus improves liver perfusion, we monitored the ability of the organ to respond to dynamic changes in the level of inspired oxygen using non-invasive MRI. Briefly, at different time points after the onset of a VEGF switch animals were anesthetized and imaged during inhalation of air and subsequently during inhalation of an oxygen-enriched (95\%) atmosphere. Changes in hemoglobin saturation were then detected by $\mathrm{T}_{2}{ }^{*}$ weighted gradient echo imaging using the intrinsic contrast generated by changes in deoxyhemoglobin content. An increased MRI signal reflects an improved capacity of erythrocytes to deliver inhaled oxygen to the tissue that, in this experimental setting, is due to increased vascularization and thus an improved perfusion (Abramovitch et al., 1998; Carmeliet et al., 1998). A recent study further validated the utility of blood oxygenation level detection (BOLD) contrast MRI for spatial mapping of vascular function through direct comparison with spectroscopic data obtained using intravital microscopy (Neeman et al., 2001). As shown in Figure 4 (right hand panels) from a representative animal, ongoing VEGF expression resulted in a progressive increase in the perfusion capability of the liver. A significant increase was already detected 11 days after the induction of VEGF and was further improved by day 20, reaching a remarkable 4-fold increase overall (for quantification see Figure 6A). As is evident from Figure 4, perfusion was improved in vast areas distributed throughout the liver. These findings demonstrate that new vessels induced by VEGF in an adult organ can indeed improve vascular function. At this time, this analysis has not been extended to the heart because taking MRI recordings from a fast beating organ requires instrumentation that is not currently available to us. 


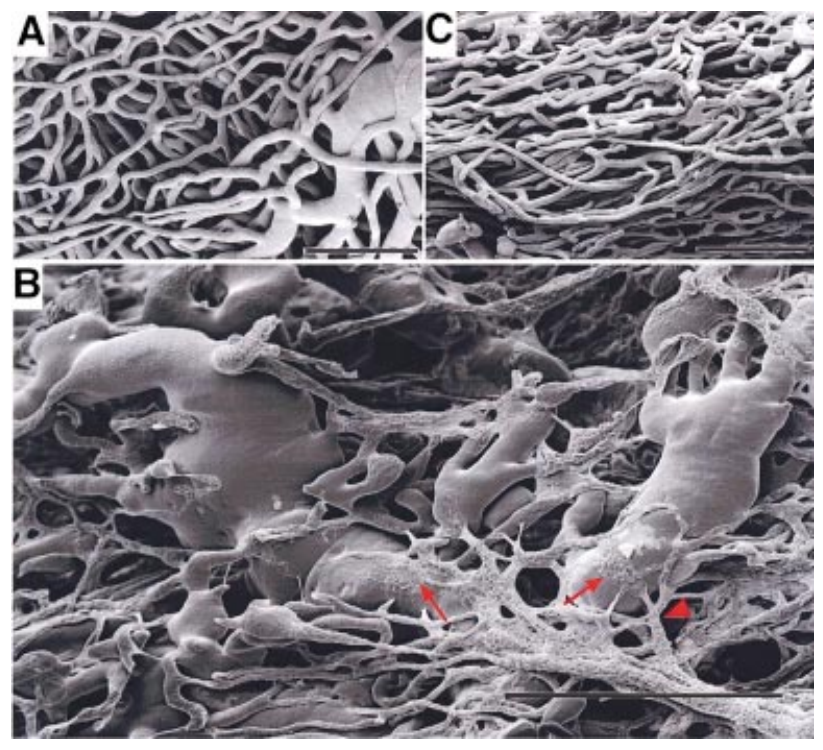

Fig. 3. Corrosion casts of epicardial vessels. Corrosion casts were prepared and scanned as described in Materials and methods. Data shown are of 10-week-old littermates. (A) Control heart. (B) Four weeks after switching 'on' VEGF. Arrows point at examples where flattened vessels merge with hemangioblastoma-like vessels. Arrowhead points at an abnormal multiple branching patterns. (C) Four weeks of 'on' followed by 8 days of 'off'. Bars represent $50 \mu \mathrm{m}$ in (A) and (C), and $100 \mu \mathrm{m}$ in (B).

MRI analysis was also useful for online monitoring of edema resulting from VEGF overexpression, as was evident from the 'whitening' of the liver due to an elevated water retention (Figure 4, left hand panels). Excessive vessel permeability and a resultant edema were evident from early on and were further aggravated by a sustained VEGF stimulus (Figures 4 and 6B). A considerable enlargement (up to 3-fold) of the edematous liver was also evident from the MRI images. This finding underscores another major problem inherent to a VEGFbased therapy, namely the need to uncouple these two activities of VEGF/vascular permeability factor (VPF).

\section{A critical duration of the VEGF stimulus is essential for preventing vessel regression and for long-term persistence of the vascular gain}

To determine whether vessels induced in an adult organ are unstable and require a continuing presence of VEGF, we took advantage of the reversibility of the tetracyclineregulated transgenic system. VEGF expression in the liver was induced and maintained for 2 weeks. Under these conditions, an elaborate network was produced that is clearly visible in sections as a network of sinusoidal vessels, giving the liver a distinct 'spongy' appearance (Figure 5A). Mice were then given tetracycline and sections were inspected 10 days later. As shown in Figure 5A, most acquired vessels have disappeared and the normal 'tight' appearance of the liver was restored. The regression of vessels as the result of VEGF withdrawal was also apparent from external inspection of superficial hepatic vessels that have almost completely disappeared where VEGF was withdrawn 2 weeks after the onset of the switch (Figure 5B). In a marked contrast, delaying the 'off' switch until 4 weeks after its onset have shown that vessels

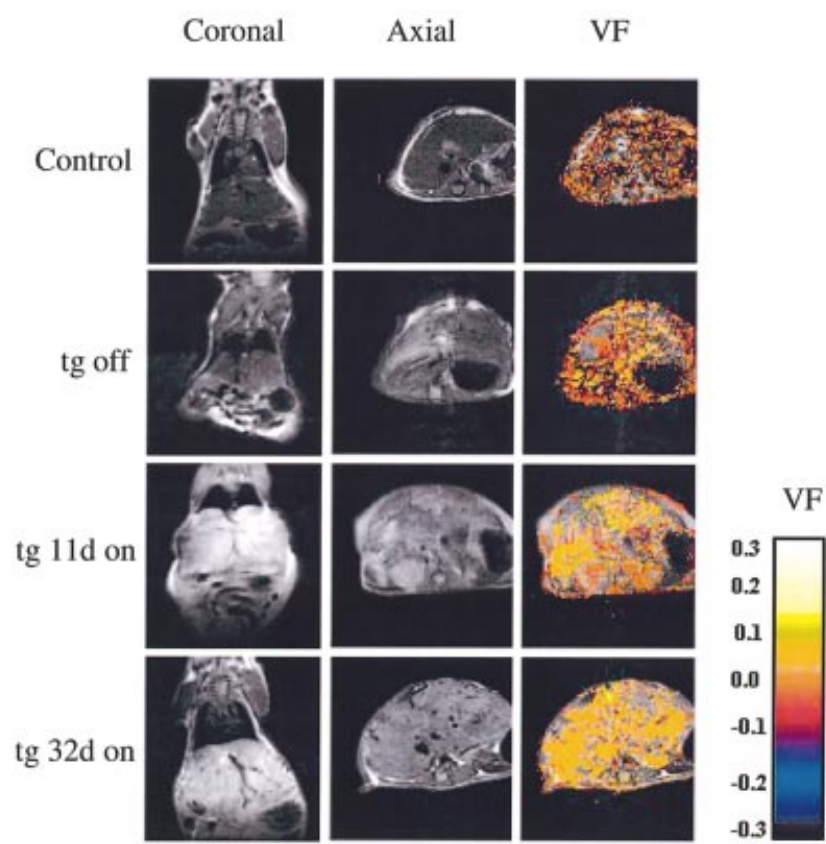

Fig. 4. MRI analysis of vascular function and permeability. Left and middle panels: MRI images of the liver. Images shown for a double transgenic animal were derived from the same coronal or axial section taken prior to ( $\mathrm{tg}$ off) or at the indicated time after switching 'on' VEGF. d, days. Note progressive whitening of the image indicative of increased water retention (i.e. edema). Also note the formation of very large vessels discernible even at this low resolution. Right panels: vascular function (VF) in the same axial section shown in the middle panels was determined as described in Materials and methods. A progressive increase in the level of oxygenation is evident, represented on a color-coded relative scale.

have become refractory to VEGF withdrawal (Figure 5B). Remarkably, under these circumstances the acquired vasculature persisted for many months after cessation of the VEGF stimulus (Figure 5C; for several examples of acquired hepatic vessels persisting 8 months after VEGF withdrawal, see Supplementary figure 2). These findings indicate that there is a critical transition point beyond which the initially unstable vessels no longer need VEGF for survival.

Similar experiments of VEGF withdrawal were also carried out in the heart. In particular, we wished to determine the nature of the vascular network that might persist after terminating the VEGF stimulus. To this end, the system was switched off after 4 weeks of a continuous VEGF stimulus and the network was visualized by corrosion casting 8 days thereafter. As can be seen in the representative cast shown in Figure $3 \mathrm{C}$, at least a fraction of induced vessels remained, as is evident by the observation of significantly increased microvessel density (compared with the ground state shown in Figure 3A). Interestingly, the abnormal vascular structures and connections produced initially (Figure $3 \mathrm{~B}$ ) seem to have disappeared. It remains to be determined whether this reflects a differential vulnerability of these vessels or the result of an extensive hemodynamic remodeling.

Of paramount importance to VEGF-based therapy is the question of whether it can yield long-term improvement of vascular function. To study this question, changes in vascular function were monitored by MRI over a period of 

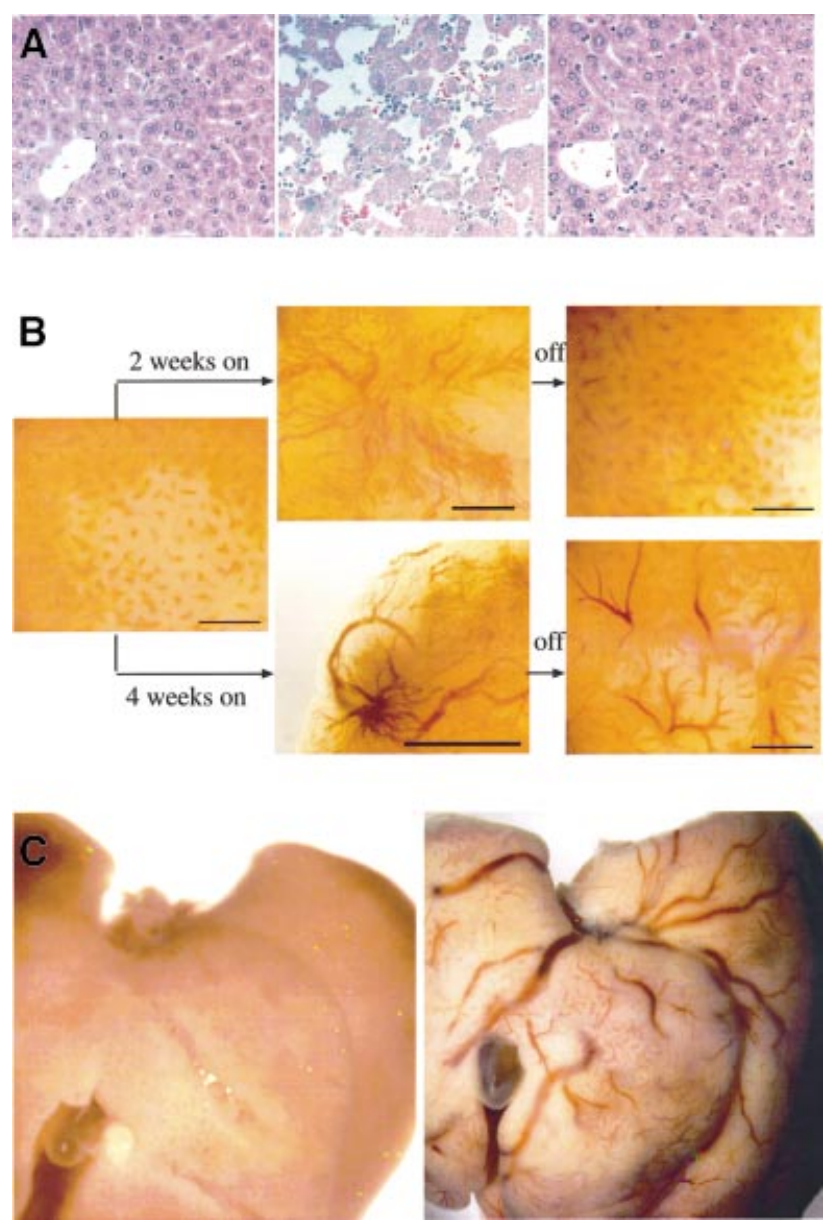

Fig. 5. Vascular phenotypes resulting from switching off VEGF. (A) Hematoxylin-eosin-stained sections of liver specimens obtained from littermate control (left), $\mathrm{tTA}^{+} / \mathrm{VEGF}^{+}$animals 14 days after switching on VEGF (middle) and from $\mathrm{tTA}^{+} / \mathrm{VEGF}^{+}$animals 14 days after switching on VEGF followed by switching off for 10 days (right). (B) Whole-mount views of livers. $\mathrm{tTA}^{+} / \mathrm{VEGF}^{+}$livers before (left) and after (middle) the indicated times of an 'on' switch, and after an additional 5 weeks of an 'off' switch (right). Bar, $1 \mathrm{~mm}$. (C) Top view of the entire liver from a control (left) and 4 months after switching off VEGF (following a 4 week stimulus).

several months during which different regimens of 'on' and 'off' cycles were examined. As already shown in Figure 4 for a single animal and further extended and quantified in experiments summarized in Figure 6A, VEGF-induced angiogenesis improved the perfusion capability of the liver 3- to 4-fold. While maximal improvement in vascular function was achieved as early as 15 days after the induction of VEGF, withdrawing VEGF at this point led to a rapid loss of all functional gain (Figure 6A, squares). In contrast, if the presence of VEGF was prolonged for an additional 15 days (anticipating that by this time the acquired vasculature should have stabilized) and then terminated, a significant fraction of the functional gain was maintained. An improved perfusion (2.5-fold) persisted for as long as 6 months after VEGF withdrawal (Figure 6A, triangles).

As noted above, VEGF-driven neovascularization was accompanied by excessive edema throughout the period where VEGF was overexpressed. Switching off VEGF expression at either schedule resulted in a rapid restoration of the normal water content in the liver (Figure 6B).
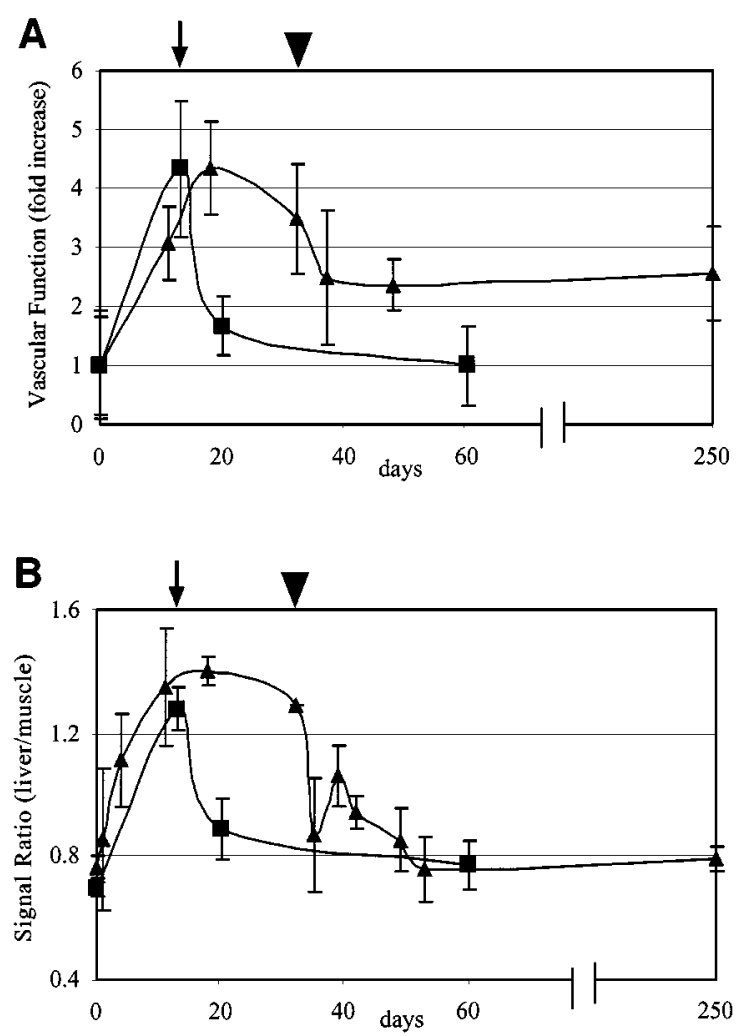

Fig. 6. Long-term improvement of vascular function in the absence of edema. Monitoring vascular function (A) and edema (B) using MRI was performed as described in the legend to Figure 4. VEGF production was induced in $\mathrm{tTA}^{+} / \mathrm{VEGF}^{+}$animal on day 0 and was switched off either at day 13 (arrow) or at day 32 (arrowhead). Each animal was imaged at the indicated times (squares, short switch; triangles, long switch) and each experimental point represents the average $\pm \mathrm{SD}$ of readings from four animals. The pre-induction reading in (A) was used as a reference for determining relative increase in liver oxygenation. For monitoring liver edema in (B), the signal intensity in the liver was standardized in each section for the signal intensity in an irrelevant tissue (muscle) whose water content was not affected by VEGF produced by the liver.

Together, these findings demonstrate that, in principle, it might indeed be possible to attain a long-term improvement in vascular function in the absence of edema. Histological inspection of livers at 8 months after cessation of VEGF induction showed that the bulk of cellular damage incurred during the period of VEGF induction and the resultant edema has been repaired (see Supplementary figure 3). Nevertheless, biochemical analysis of blood specimens obtained from these animals indicated a chronic mild hepatocellular damage (reflected in elevated levels of alanyl-amino transferase averaging $150 \mu / 1$, compared with $47 \mu / 1$ in controls). No long-term cholestatic damage was detected (reflected in no significant change in blood levels of alkaline phosphatase or $\gamma$-glutamyl transferase). These findings suggest that excessive edema associated with surplus VEGF might cause a mild long-term liver damage.

\section{Discussion}

This study was undertaken to examine the architecture and functionality of vascular networks induced by VEGF in adult organs. This is a situation faced in an attempted 
pro-angiogenic therapy where VEGF, unassisted by other exogenous factors, is expected to induce new vessels that will properly connect to the pre-existing network conferring a long-term improvement of organ perfusion. While of particular relevance to therapeutic angiogenesis, a major objective of this study was to shed light on the mechanism of adult neovascularization, in general, reasoning that our poor understanding of the process might impede ongoing clinical trials. To this end we devised a genetic switch model system that enabled us to set adult neovascularization in motion and thereby uncover inherent problems of VEGF-based therapy in a manner independent of compounding factors associated with any particular treatment modality.

New vessels continued to be formed as long as the inductive VEGF stimulus was present, and a prolonged stimulus resulted in an exaggerated angiogenic response to the extent that new vessels destroyed the normal organ architecture. This finding demonstrates the lack of an effective negative feedback control operating to limit VEGF-driven neovascularization in an adult organ. This is in contrast to other processes of adult neovascularization, such as the one associated with wound repair or periodic neovascularization in the ovary and endometrium, which are self-limiting processes. Thus, it appears that downregulation of the inducing VEGF stimulus is the major mechanism preventing excessive neovascularization in the adult, rather than angiogenic inhibitors coming into play or the exhaustion of a particular downstream effector molecule. These findings might also explain why a precise VEGF dosage is so critical for a balanced vascular development.

A fundamental problem with forcing formation of new blood vessels in an adult organ stems from the lack of spatial cues that will direct newly formed vessels in the process of their connection with the existing coronary network. In this respect, it differs from embryonic neovascularization, where stereo-specific guiding cues do exist, from arterialization, where hemodynamic changes within the existing network determine the sites of an interstitial increase in vessel diameter, and also from the situation in tumors which are vascularized de novo. It remained to be determined whether a relatively uniform field of VEGF instructs the formation of a properly graded vascular tree that is appropriately integrated into existing arterial and venous branches. Notably, even in the absence of precise dosage and spatial and temporal regulations, a reasonable degree of vascular morphogenesis still took place, preserving the basic parameters of connecting to the systemic circulation, forming a highly branched vascular tree and acquisition of a periendothelial cell coating. Thus, VEGF seems to play a more permissive than instructive role in adult neovascularization. In contrast, other morphogenetic characteristics of developmental neovascularization, such as a stereospecific branching pattern and a proper gradation in vessel diameters were not maintained. High-power visualization of the continuum of perfused vessels has demonstrated abnormal connections and branching patterns in the induced coronary network that might be difficult to reconcile with an orderly flow. Acquired vascular loops did not form the normal pattern of a graded vascular tree; in many instances a single vessel appeared to contain a non-uniform lumen size and in extreme cases sac-like structures were produced. Judging from the vascular casts, it appears that VEGF-mediated hyperfusion may play a role in the generation of glumeroloid and hemangioma-like vessels, similar to those previously encountered in other settings of VEGF overexpression (Drake and Little, 1995; Springer et al., 1998; Sundberg et al., 2001). In light of this finding it was somewhat surprising that the induced network conferred a long-term improvement of organ perfusion. We propose that an extensive remodeling plays a major role in reshaping the initial disorganized system into an efficient circulatory network. Supporting this possibility was our observation that when examined several weeks and even months after VEGF withdrawal, the induced network appeared more organized.

Single factor therapy is based on the premise that the factor employed can orchestrate the entire angiogenic cascade and induce all other activities required collectively for the formation of a functional vascular network. Using a measurable gain in vascular function as an endpoint, the data provided here clearly show that this is indeed the case for a VEGF-initiated process. Importantly, the use of the conditional expression system enabled us to demonstrate that VEGF can promote the entire angiogenic cascade in the absence of ischemia or inflammation. This finding runs counter to the notion that the non-ischemic myocardium might not be responsive to VEGF and that additional hypoxia-inducible factors might be essential. Our findings also challenge the notion that inflammation is a mandatory requirement for efficient myocardial neovascularization. It is often assumed that ageing adult organs will be less responsive to angiogenic stimuli. While this issue was not directly addressed by this study, we note that a robust angiogenic response was induced even when the VEGF switch was delayed until 4.5 months after birth (data not shown), arguing that the fully mature organ can still respond to exogenous VEGF.

The results reported here clearly show that VEGF levels sufficient for induction of new vessels also cause severe tissue edema. It might therefore be essential to counteract the permeabilizing activity of VEGF, particularly with long-term treatment. Since the edema is resolved shortly after terminating VEGF, the need to prevent excessive vascular leak might be transient (i.e. until vessels become refractory to VEGF) and, therefore, therapeutically feasible. Recent studies have suggested at least two ways to selectively inhibit excessive leakage of VEGF-induced vessels: through the use of $s r c$ inhibitors (Eliceiri et al., 1999) or through the action of co-expressed Ang-1 (Thurston et al., 1999).

The elaborate vascular networks induced by VEGF in the heart and liver almost completely disappeared upon early termination of the inductive signal, and this was also reflected in a full reversal of the net gain in vascular function. However, with longer duration of the VEGF stimulus we observed long lasting, VEGF-independent vessels. The molecular and cellular determinants of the transition from a VEGF-dependent to a VEGF refractory state remain to be determined. Our previous studies in the retina and tumor systems have suggested that the recruitment of periendothelial cells is the critical event marking vessel maturation (Benjamin et al., 1998). Ongoing studies are examining whether the engagement with periendo- 
thelial cells is also a stabilizing determinant in the case of adult myocardial angiogenesis. Irrespective of the mechanism, the finding of an obligatory requirement for a relatively long presence ( $>2$ weeks) of VEGF to produce durable vessels is of fundamental importance in ongoing debates regarding the preferred mode of pro-angiogenic therapy. Thus, modalities relying on a transient presence of VEGF in the target tissue (e.g. adenovirus based vectors with rapid clearance) are unlikely to produce durable vessels. Clearly, the consensus that vectors with a short (5-7 days) duration of expression are highly desirable (Simons et al., 2000) needs to be re-considered. Interestingly, whereas 14 days of an 'on' switch produced nondurable vessels, a protocol of two 14-day 'on' episodes separated by 5 weeks of an 'off' switch produced stable vessels persisting for months without VEGF (data not shown). Again, this finding should be taken into account when weighing the potential benefits of a single versus repeated treatments or a sustained delivery.

In conclusion, this study has demonstrated the promise of a VEGF-based therapy but also revealed some inherent obstacles that need to be resolved prior to its implementation.

\section{Materials and methods}

\section{Transgenic mice}

The pTET-VEGF164 responder line was as described previously (Dor et al., 2001). The MHC $\alpha$-tTA heart-specific driver line (Yu et al., 1996) and the $\mathrm{P}_{\mathrm{LAP}}$-tTA liver-specific driver line (Kistner et al., 1996; Lavon et al., 2000) were as described previously. Crosses were carried out between pTET-VEGF ${ }^{+-}$and the respective $\mathrm{tTA}^{+/-}$driver line. Offspring were genotyped by PCR. Primers for PCR genotyping of the pTETVEGF164 transgene have been described previously (Benjamin and Keshet, 1997). The tTA transgene was amplified using primers 5'-TAGATGTGCTTTACTAAGTCATCGCG and 5'-GAGATCGAGCAGGCCCTCGATGGTAG. Littermates that inherited none or only one transgene served as controls. To repress pTET-VEGF164 during embryonic and early postnatal heart and liver development mothers were given $500 \mu \mathrm{g} / \mathrm{ml}$ tetracycline (Teva Inc., Israel)/3\% sucrose in the drinking water throughout gestation and lactation periods.

\section{VEGF expression analysis}

In situ hybridization was performed on paraffin sections as described previously (Motro et al., 1990) using VEGF and tTA-specific probes (Dor et al., 2001).

Quantification of in situ hybridized VEGF was carried-out as follows: $3-\mu \mathrm{m}$ thick sections were hybridized with a VEGF-specific probe encompassing the entire coding sequence. Brightfield and darkfield images $(\times 100)$ were captured using a digital camera (SV-Micro Inc.) and stored as Adobe photoshop files. Darkfield images were converted in such a way that autoradiographic grains are visualized as discrete black dots. NIH-IMAGE software was used to count grains representing VEGF signal. Relative grain densities in the control/transgenic heart or liver were determined in three thin sections of each of three independent organs.

Relative VEGF mRNA levels were also quantified by densitometric tracing of northern blots as follows: total RNA was extracted from the respective organ using TRI reagent (Sigma) according to the manufacturer's instructions, and $5 \mu \mathrm{g}$ resolved on agarose gel. Following blotting, membranes were hybridized with a ${ }^{32} \mathrm{P}$-labeled probe detecting only the VEGF coding region (thus detecting endogenous and transgenic VEGF with the same efficiency). The difference in transcript size between endogenous and transgenic VEGF (3.7 and $\sim 2 \mathrm{~kb}$, respectively) allowed independent scanning of the respective autoradiographic bands and quantification of band intensities (relative to ribosomal RNA) using NIH image software.

VEGF protein levels in sera of mice were measured using a mouse VEGF ELISA kit (Oncogene Inc.) according to the manufacturer's instructions.

\section{Corrosion casting}

Vascular casts were prepared as described previously (Djonov et al., 2000). Briefly, mice were anesthetized using an overdose of pentobarbitol, and mercox solution (Vilene Co., Japan) containing $0.1 \mathrm{ml}$ accelerator per $5 \mathrm{ml}$ resin was injected into the left ventricle. Following dissolution of tissue in $15 \% \mathrm{KOH}$ for 6 weeks, casts were dehydrated in ethanol and dried in a vacuum desiccator. Specimens were glued onto stubs with carbon and spattered with gold, and examined using a Philips XL 30 FEG scanning electron microscope.

\section{MRI}

MRI experiments were performed on a horizontal 4.7 T Bruker Biospec spectrometer (Bruker, Germany) using volume coil. Mice were anesthetized, placed supine with the liver located at the center of the coil and scanned twice a week. Spin echo images were used to evaluate the relative water content of the tissue $[\mathrm{TR} / \mathrm{TE}=2000 / 20 \mathrm{~ms}$; field of view $($ FOV $)=4 \times 4 \mathrm{~cm}$; slice thickness of $1 \mathrm{~mm} ; 256 \times 256$ matrix $]$. Mean signal intensity was calculated for the liver and was normalized to the signal intensity from the muscle. This ratio prior to switching on VEGF was $\sim 0.7$ and reached values of up to 1.5 following the switch.

Functionality of the vasculature was determined from $\mathrm{T}_{2} *$-weighted gradient echo images acquired during inhalation of air- $\mathrm{CO}_{2}(95 \%$ air and $5 \% \mathrm{CO}_{2}$ ), and oxygen- $\mathrm{CO}_{2}$ (95\% oxygen and $5 \% \mathrm{CO}_{2} ;$ carbogen) as described previously (Abramovitch et al., 1998). The different gas mixtures were administered to the mice via a home-made mask. Four images were acquired at each gas mixture $(51 \mathrm{~s} / \mathrm{image}$; slice thickness $1 \mathrm{~mm}$; TR $=100 \mathrm{~ms}$; TE $=130 \mathrm{~ms}$; spectral width $25000 \mathrm{~Hz}$; FOV $3 \mathrm{~cm}$; $256 \times 256$ pixels; in plane resolution $110 \mu \mathrm{m}$; four averages). MRI data were analyzed using IDL software (Research Systems Inc., CO). Vascular function (VF) was derived from images acquired during inhalation of carbogen and air- $-\mathrm{CO}_{2}$ using the following equation:

$$
\mathrm{VF}=\mathrm{b} \Delta \mathrm{Y}=\frac{\ln \left(\mathrm{I}_{\text {carbogen }} / \mathrm{I}_{\text {air- }}-\mathrm{CO}_{2}\right)}{\mathrm{TE} \times \mathrm{C}_{\mathrm{MRI}}}
$$

where I is the mean signal intensity in the liver during inhalation of either carbogen or air- $\mathrm{CO}_{2}, \mathrm{TE}$ is the echo time, $\mathrm{Y}$ is the fraction of oxyhemoglobin, $\mathrm{b}$ is the volume fraction of blood and $\mathrm{C}_{\mathrm{MRI}}=599 / \mathrm{s}$ at 4.7 T. This parameter measures the capacity of oxygen delivery from the lungs to each pixel in the image (Abramovitch et al., 1998; Carmeliet et al., 1998). Data are presented as color map overlaid on the gray scale baseline image for values of $\mathrm{VF}>0.005$.

\section{Supplementary data}

Supplementary data for this paper are available at The EMBO Journal Online.

\section{Acknowledgements}

The authors thank Dr H.Bujard for providing the $\mathrm{P}_{\mathrm{LAP}}-\mathrm{tTA}$ transgenic mice and the Arthur and Rochelle Belfer foundation for financial support.

\section{References}

Abramovitch,R., Frenkiel,D. and Neeman,M. (1998) Analysis of subcutaneous angiogenesis by gradient echo magnetic resonance imaging. Magn. Reson. Med., 39, 813-824.

Adams,R.H. and Klein,R. (2000) Eph receptors and ephrin ligands. Essential mediators of vascular development. Trends Cardiovasc. Med., 10, 183-188.

Alon,T., Hemo,I., Itin,A., Pe'er,J., Stone,J. and Keshet,E. (1995) Vascular endothelial growth factor acts as a survival factor for newly formed retinal vessels and has implications for retinopathy of prematurity. Nature Med., 1, 1024-1028.

Benjamin,L.E. and Keshet,E. (1997) Conditional switching of vascular endothelial growth factor (VEGF) expression in tumors: induction of endothelial cell shedding and regression of hemangioblastoma-like vessels by VEGF withdrawal. Proc. Natl Acad. Sci. USA, 94, 8761-8766.

Benjamin,L.E., Hemo,I. and Keshet,E. (1998) A plasticity window for blood vessel remodelling is defined by pericyte coverage of the preformed endothelial network and is regulated by PDGF-B and VEGF. Development, 125, 1591-1598. 
Blau,H.M. and Banfi,A. (2001) The well-tempered vessel. Nature Med., 7, 532-534.

Carmeliet,P. (2000) VEGF gene therapy: stimulating angiogenesis or angioma-genesis? Nature Med., 6, 1102-1103.

Carmeliet,P. et al. (1996) Abnormal blood vessel development and lethality in embryos lacking a single VEGF allele. Nature, 380, 435-439.

Carmeliet,P. et al. (1998) Role of HIF-1 $\alpha$ in hypoxia-mediated apoptosis, cell proliferation and tumor angiogenesis. Nature, 394, 485-490.

Djonov,V., Schmid,M., Tschanz,S.A. and Burri,P.H. (2000) Intussusceptive angiogenesis: its role in embryonic vascular network formation. Circ. Res., 86, 286-292.

Dor,Y. and Keshet,E. (1997) Ischemia-driven angiogenesis. Trends Cardiovasc. Med., 7, 289-294.

Dor,Y., Gamenisch,T.D., Itin,A., Fishman,G.L., Mcdonald,J.A., Carmeliet,P. and Keshet,E. (2001) A novel role for VEGF in endocardial cushion formation and its potential contribution to congenital heart defects. Development, 128, 1531-1538.

Drake,C.J. and Little,C.D. (1995) Exogenous vascular endothelial growth factor induces malformed and hyperfused vessels during embryonic neovascularization. Proc. Natl Acad. Sci. USA, 92, 7657-7661.

Eliceiri,B.P., Paul,R., Schwartzberg,P.L., Hood,J.D., Leng,J. and Cheresh,D.A. (1999) Selective requirement for Src kinases during VEGF-induced angiogenesis and vascular permeability. Mol. Cell, 4, 915-924.

Ferrara,N., Carver Moore,K., Chen,H., Dowd,M., Lu,L., O'Shea,K.S., Powell Braxton,L., Hillan,K.J. and Moore,M.W. (1996) Heterozygous embryonic lethality induced by targeted inactivation of the VEGF gene. Nature, 380, 439-442.

Freedman,S. and Isner,J.M. (2001) Therapeutic angiogenesis for ischemic cardiovascular disease. J. Mol. Cell. Cardiol., 33, 379-393.

Gale,N.W. and Yancopoulos,G.D. (1999) Growth factors acting via endothelial cell-specific receptor tyrosine kinases: VEGFs, angiopoietins and ephrins in vascular development. Genes Dev., 13, $1055-1066$.

Hammond,H.K. and McKirnan,M.D. (2000) Angiogenic gene therapy for heart disease: a review of animal studies and clinical trials. Cardiovasc. Res., 49, 561-567.

Helisch,A. and Schaper,W. (2000) Angiogenesis and arteriogenesis-not yet for prescription. Z. Kardiol., 89, 239-244.

Kistner,A., Gossen,M., Zimmermann,F., Jerecic,J., Ullmer,C., Lubbert,H. and Bujard,H. (1996) Doxycycline-mediated quantitative and tissue-specific control of gene expression in transgenic mice. Proc. Natl Acad. Sci. USA, 93, 10933-10938.

Laham,R.J., Simons,M. and Sellke,F. (2001) Gene transfer for angiogenesis in coronary artery disease. Annu. Rev. Med., 52, $485-502$.

Lavon,I. et al. (2000) High susceptibility to bacterial infection, but no liver dysfunction, in mice compromised for hepatocyte NF- $\mathrm{KB}$ activation. Nature Med., 6, 573-577.

Lee,R.J., Springer,M.L., Blanco-Bose,W.E., Shaw,R., Ursell,P.C. and Blau,H. (2000) VEGF gene delivery in the myocardium: deleterious effects of unregulated expression. Circulation, 102, 898-901.

Lindahl,P., Johansson,B.R., Leveen,P. and Betsholtz,C. (1997) Pericyte loss and microaneurism formation in PDGF-B-deficient mice. Science, 277, 242-245.

Miquerol,L., Langille,B.L. and Nagy,A. (2000) Embryonic development is disrupted by modest increases in vascular endothelial growth factor gene expression. Development, 127, 3941-3946.

Motro,B., Itin,A., Sachs,L. and Keshet,E. (1990) Pattern of interleukin 6 gene expression in vivo suggests a role for this cytokine in angiogenesis. Proc. Natl Acad. Sci. USA, 87, 3092-3096.

Neeman,M., Dafni,H., Bukhari,O., Braun,R.D. and Dewhirst,M.W. (2001) In vivo BOLD contrast MRI mapping of subcutaneous vascular function and maturation: validation by intravital microscopy. Magn. Reson. Med., 45, 887-898.

Simons,M. et al. (2000) Clinical trials in coronary angiogenesis: issues, problems, consensus: an expert panel summary. Circulation, 102, E73-E86.

Springer,M.L., Chen,A.S., Kraft,P.E., Bednarski,M. and Blau,H.M. (1998) VEGF gene delivery to muscle: potential role of vasculogenesis in adults. Mol. Cell, 2, 549-558.

Sundberg,C., Nagy,J.A., Brown,L.F., Feng,D., Elckelhoefer,I.A., Manseau,E.J., Dvorak,A.M. and Dvorak,H.F. (2001) Glumeroid microvascular proliferation follows adenoviral vascular permeability factor/vascular endothelial growth factor-164 gene delivery. Am. $J$. Pathol., 158, 1145-1160.

Thurston,G., Suri,C., Smith,K., McClain,J., Sato,T.N., Yancopoulos, G.D. and McDonald,D.M. (1999) Leakage-resistant blood vessels in mice transgenically overexpressing angiopoietin-1. Science, 286, 2511-2514.

van Royen,N., Piek,J.J., Buschmann,I., Hoefer,I., Voskuil,M. and Schaper,W. (2001) Stimulation of arteriogenesis; a new concept for the treatment of arterial occlusive disease. Cardiovasc. Res., 49, 543-553.

Yu,Z., Redfern,C.S. and Fishman,G.I. (1996) Conditional transgene expression in the heart. Circ. Res., 79, 691-697.

Received November 13, 2001; revised February 15, 2002; accepted February 20, 2002 